\title{
TEMIAR WOMEN BREASTFEEDING EXPERIENCES: A JOURNEY TO EMPOWERMENT
}

\author{
Sharifah Zahhura Syed Abdullah ${ }^{1^{*}}$ and Rozieyati Mohamed Saleh ${ }^{2}$ \\ ${ }^{1}$ Centre for Research on Women and Gender (KANITA), Universiti Sains Malaysia, \\ Pulau Pinang, MALAYSIA \\ ${ }^{2}$ School of Health Sciences, Universiti Sains Malaysia, Kubang Kerian, Kelantan, \\ MALAYSIA
}

*Corresponding author: zahhura@usm.my

Published online: 15 April 2020

To cite this article: Sharifah Zahhura Syed Abdullah and Rozieyati Mohamed Saleh. 2020. Temiar women breastfeeding experiences: A journey to empowerment. Kajian Malaysia 38(Supp.1): 133-143. https://doi.org/10.21315/km2020.38.s1.8

To link to this article: $\mathrm{https} / / /$ doi.org/10.21315/km2020.38.s1.8

\begin{abstract}
Empowerment of women pertaining to breastfeeding gives women the ability to act and the right to do so. Breastfeeding confirms a woman's power to control her own body and a woman's unique ability to care for her infant in the best way possible. Empowerment of mothers will help protect or restore breastfeeding cultures around the world. A qualitative research method involving 15 in-depth interviews and four focus group discussions were chosen in this study to compare and contrast two different locations: the communities of Pos Tuel and Pos Brooke in Kelantan, Malaysia which represent different lifestyle experiences of indigenous Temiar people. Analysis of participants' descriptions led to the emergence of four main categories: knowledge and skills pertaining to breastfeeding, maternal satisfaction and enjoyment in breastfeeding, family belief in the value of breastfeeding, and breastfeeding support from surrounding people. The findings from this study are crucial in the preservation of breastfeeding culture among the women and can be used to design comprehensive plans in promoting breastfeeding among other indigenous groups.
\end{abstract}

Keywords: empowerment, breastfeeding, health, Temiar women 


\section{INTRODUCTION}

The World Health Organisation (WHO) advises that all infants be exclusively breastfed in the first six months and continue to be breastfed until they reach two years of age or longer, along with complementary foods (WHO 2001). The advantages of breastfeeding for both mothers and babies are numerous, inclusive of long-term and short-term health benefits.

Breastfeeding is a woman's right. It is an important woman's issue, human rights issue, and feminist issue since breastfeeding empowers women and contributes to gender equality. Breastfeeding encourages women's self-reliance by increasing their confidence in their ability to meet the needs of their infants (Van Esterik n.d.). Empowerment means the ability of people to gain understanding and control over personal, social, economic and political forces to take action to improve their lives; the range of activities from individual self-assertion to collective resistance, protest and mobilisation that challenge power relations and to make (someone) stronger and more confident, especially in controlling their lives and claiming their rights (World Alliance for Breastfeeding Action 1995). Empowerment, as adopted by Ketabi, Yazdkhasti and Farohki (2003) is a process by which women become aware of their needs and demands and reinforce the courage in them to reach their goals and develop the abilities required for actualising their demands.

According to Kohan, Heidari and Keshvari (2016), one of the important factors that influence a mother's decision to breastfeed and to continue it, is the mother's empowerment in breastfeeding. A study by Kang, Choi and Ryu (2008) also showed that empowerment of women increases breastfeeding rates and helps mothers in surviving and coping with breastfeeding problems and difficulties. Breastfeeding can empower women by reducing a mother's economic and medical dependence. It diminishes the power of commercial interests to manipulate the advertising of breast milk substitutes, confirms a woman's power to control her own body - breastfeeding challenges the established medical models and business interests that promote bottle feeding, confirms a woman's unique ability to care for her infant in the best way possible, challenges the view of breasts as merely sex objects and promotes optimum infant and maternal health (World Alliance for Breastfeeding Action 1995).

Many studies have shown that family is the main influence on breastfeeding and family members were more influential than professionals (Mitchell-Box and Braun 2013; Yang et al. 2016; Abbass-Dick 2013). Family influence is particularly salient where decision making is more of a cooperative effort, such as in many developing countries. Support from family is critical for the success of breastfeeding, if fathers and family members are supportive of 
breastfeeding, the mother is more likely to initiate breastfeeding and to breastfeed for longer. Abbass-Dick and colleagues (2018) noted that breastfeeding decisions are so deeply embedded in family decision-making that they adopted the term "breastfeeding teamwork" to describe it. Breastfeeding teamwork includes shared responsibilities and increased collective involvement in child-rearing. Their research also points out that effective breastfeeding support by a father includes the ability to be responsive to the mother's needs, including respecting her autonomy when she does not actually need help. This also reflects that the involvement of fathers and men in the support of breastfeeding is woman-centric and empowers her within a family and community.

Omer-Salim (2018) has suggested that the empowerment of women can be achieved by promoting, protecting and supporting breastfeeding in rural communities. The rural communities can be collectively empowered to recognise that breastfeeding plays a particularly important role in the health and well-being of families. This means that breastfeeding needs to be supported by everyone in the community, including men and boys. Hormonal and neurobiological changes happen in both men and women and can result in more nurturing behaviours toward their child if they are supported to do so. Mother's breastfeeding empowerment is a key motivational, psychological and changeable factor for continuous breastfeeding. Successful breastfeeding depends on various physiological and psychological factors in a mother (Heidari, Keshvari and Kohan 2016).

Orang Asli Temiar are one of the indigenous peoples of the peninsula. Once, the Temiar could only be found scattered in the remote forests. Nowadays, although most still live a traditional life or a tradition-bound lifestyle, others have moved out of the forest to settle in more accessible locales. Some live in villages and some have migrated to towns and cities. Today, the Temiar is changing as contact with the Malays and other groups in the population intensify. As their lifestyles change, so does their culture. Yet, treating culture as an abstraction may lead one to ignore the basic human rights of small-scale societies and ethnic minorities to maintain their cultural heritage in the face of threats from dominant societies (Bodley 1994). Even though breastfeeding is often described as "natural", being exposed and manipulated by others on the importance of bottle or formula feeding might influence the Temiar mothers in adapting to this belief given their vulnerability in this particular issue. Empowerment in breastfeeding plays a key role in breastfeeding promotion such that exclusive breastfeeding increases with an enhanced level of breastfeeding empowerment, therefore, this study with a qualitative approach was designed and carried out aiming to explore the Temiar women's experiences and understanding pertaining to empowerment in breastfeeding. 


\section{METHODOLOGY}

The present study employed a qualitative design using in-depth interviews and focus group discussions. Qualitative methods allow the researcher to generate a detailed understanding of experiences (Bazeley 2007; Munhall 2011). A total of 15 interviews and 4 focus group discussions were carried out. In-depth interviews were conducted face to face. No repeat interviews were undertaken. The recordings were transcribed verbatim. Participants were asked a series of semi-structured questions. Prompts and follow-up questions were used to obtain further narratives.

Focus group discussions consisted of the older and young cohorts of mothers. A list of criteria was drawn up and verified by each potential participant just before the group convened to ensure validity. Participants are those individuals who had been involved with breastfeeding practices. For example, women in the various households who are responsible for feeding the infants and also women who are indirectly involved in the feeding practices. All interviews and focus group discussions took place in a familiar setting in order to motivate participants and to create a comfortable environment. Each session was audio-taped and videorecorded to allow the facilitator to focus on the group responses and non-verbal behaviour and for later transcription.

A thematic approach was considered appropriate to manage the dataset and interpret the meaning of participants' breastfeeding experiences. Table 1 details the stages of the thematic analysis. Coding was a process of recognising bits and pieces of information in the transcripts and linking these to concepts and themes. Coding is an integral part of data analysis and allows a researcher to quickly retrieve relevant parts of it for discussion purposes. The researcher slowly reads the interviews and focus group transcripts, looking for themes. The themes are partly emergent and partly influenced by research aims, research questions, concepts in the literature and terms used by participants or members in the social setting. The qualitative data analysis software NVivo, version 10 (QSR International Pty Ltd, Melbourne, VIC, Australia) was used for data management. Data were reviewed until saturation and no new themes emerged. 
Table 1: Framework analysis

\begin{tabular}{|c|c|}
\hline Data management & $\begin{array}{l}\text { - Interviews are transcribed verbatim. } \\
\text { - } \text { weading and re-reading the transcribed interview allows familiarisation } \\
\text { - Key themes and minor themes are identified and coded. } \\
\text { - Initial themes are arranged into a coding index. } \\
\text { - Interview data is assigned to a theme. }\end{array}$ \\
\hline $\begin{array}{l}\text { Descriptive } \\
\text { accounts }\end{array}$ & $\begin{array}{l}\text { - Summarising and combining the range and diversity of coded data by } \\
\text { refining themes. } \\
\text { - Identifying key dimensions of the synthesised data. } \\
\text { - Developing a description of the main themes within the whole picture. }\end{array}$ \\
\hline $\begin{array}{l}\text { Explanatory } \\
\text { accounts }\end{array}$ & $\begin{array}{l}\text { - Identify and develop associations/patterns within concepts and themes. } \\
\text { - Reflect back on the original data as a whole and analytical stages in } \\
\text { order to ensure the perceptions of participants are accurately reflected } \\
\text { and reduce the possibility of misinterpretation. } \\
\text { - Interpret/find meaning and explain the concepts, themes and categories. } \\
\text { - Seek wider application of concepts and themes informed by research } \\
\text { literature. }\end{array}$ \\
\hline
\end{tabular}

Source: Morris et al. (2018).

\section{ETHICAL CONSIDERATIONS}

The research procedures were fully approved by the Human Research Ethics Committee of Universiti Sains Malaysia (FWA Reg. No.: 00007718; IRB Reg. No.: IRB00004494).

\section{FINDINGS}

A total of 15 individuals took part in one-to-one interviews and 27 mothers engage in 4 focus group discussions. Four themes were identified: (1) knowledge and skills pertaining to breastfeeding, (2) maternal satisfaction and enjoyment in breastfeeding, (3) family belief in the value of breastfeeding, and (4) breastfeeding support from surrounding people.

\section{Knowledge and Skills Pertaining to Breastfeeding}

Most of the participants in Pos Brooke believed that they had the skills and knowledge to prevent and solve breastfeeding problems. One participant said "I have breast engorgement problem during the early postpartum period but now not anymore. I used to express the milk into the bottle" (Zarina, 30 years old, Pos Brooke). However, only one participant from Pos Tuel mentioned that 
"I started breastfeeding my baby after three days...because there is no milk... need to eat nutritious food...hahaha...for example drinking...there are people selling D'Herbs...aa the delivery set...aaa...I consumed that then the milk comes out...hehe..." (Halimaton, 27 years old). Other participants from Pos Tuel agreed that they did not have any breastfeeding problems which called for further consultation.

Nevertheless, there are some participants who were having difficulties with breastfeeding practices since they were first time mothers but with the help of health practitioners, they were able to breastfeed their babies.

I do not know how but the nurse taught me... at first, she told me but I don't know...but she kept on teaching... show me how to breastfeed... then I try... at first, I don't know...the milk did not come out for two days...emmm...but I kept on breastfeeding my child...hurm...even there is no milk, I kept on giving until finally, the milk comes out... (Suzy, 23 years old, Pos Brooke)

It is difficult because the milk did not come out...two to three days like that...then I started to express...aaa the nurse and the doctor taught me... they ask me to put some hot water on the cloth then put it over the nipple. (Elina, 25 years old, Pos Brooke)

She (nurse) ask me to breastfeed...at first I did not know how...then she showed me how...mmm...hehe...she showed me how to hold the baby... before this I don't know, I hold like this (showing how she holds the baby before)...she asked me to hold the baby like this to breastfeed the baby (showing how to breastfeed the baby). (Aminah, 20 years old, Pos Brooke)

\section{Maternal Satisfaction and Enjoyment in Breastfeeding}

Most of the participants' statements showed that mothers are feeling satisfied with themselves for breastfeeding babies. Ani, a 36-year-old mother from Pos Brooke mentioned that "he is feeding nicely...he is happy...when I breastfeed him...he looks at my face and then he smiled". While Angah, 28-years-old from Pos Brooke stated that "mmm I feel happy...the baby is healthy...I gave him breast milk and he is healthy...hehehe". Other participants from Pos Tuel agreed that they feel happy with themselves such as Bedah, 34-years-old said, "I feel good...feel that she is growing faster", and Gayah, 26-year-old mentioned that, "I feel happy...I can give the breast milk to my baby". 


\section{Family Belief in the Value of Breastfeeding}

Participants mentioned that family belief in the value of breastfeeding is an encouraging factor in the empowerment of breastfeeding. When spouses and family members believe in breastfeeding and emphasise it, the mother is encouraged and feels empowered:

Like myself, my mother was my midwife for my first baby... I do not know anything, she taught me...aa...she taught...she said if I want to breastfeed the baby... after delivery... wash the nipple first...wash...then can start to breastfeed... (Sarimah, 20 years old, Pos Brooke)

aa...my mother and husband said breast milk is good for the baby... hahahaha... (Zainab, 30 years old, Pos Tuel)

\section{Breastfeeding Support from Surrounding People}

Within households, women often work together to share childcare and other responsibilities. Other family members can play a useful role in assisting new mothers by providing advice on managing to breastfeed and helping with household tasks. In the participants' view, receiving assistance from people surrounding them facilitates the breastfeeding process. A 25 -year-old mother from Pos Brooke said, "Nurse, doctor...mother...aaa my mother had taught me... if I meet my friend also...they told me...aaa they encourage me...they said I should give breast milk because it is good for the baby." Another participant said, "my mother-in-law told me what to eat to increase milk production... she asked me to eat porridge... rice porridge...hehe..." (Aminah, 20 years old, Pos Brooke). Other participants' excerpts are as follows:

My friends...nurses if we go to the clinic. (Sarimah, 20 years old, Pos Brooke)

Sometimes I asked my mother if I have breastfeeding problem. (Suzy, 23 years old, Pos Brooke)

My own mother...and my mother-in-law...they support this breastfeeding. (Bedah, 34 years old, Pos Tuel)

Sometimes my husband...if I am sick and tired...he will help... sometimes my mom. (Karimah, 26 years old, Pos Tuel)

My sister...mom...they will help to hold the baby...they will massage. (Alang, 23 years old, Pos Tuel) 
My sister...mom...they asked me to breastfeed. (Gayah, 26 years old, Pos Tuel)

My husband...he asked me to breastfeed...haha... (Halimaton, 27 years old, Pos Tuel)

\section{DISCUSSION}

The participants of this study described how they viewed empowerment in breastfeeding in four aspects: knowledge and skills pertaining to breastfeeding, maternal satisfaction and enjoyment in breastfeeding, family belief in the value of breastfeeding, and breastfeeding support from surrounding people.

The findings of the present study indicate that adequate knowledge and skills for breastfeeding practices have a positive effect on the formation of breastfeeding empowerment. This result was similar to a study by Heidari, Kohan and Keshvari (2017) which also showed that the participants' knowledge and skills to prevent and solve breastfeeding problems, and self-efficacy in solving breastfeeding problems are some of the significant factors of empowerment in breastfeeding. A study by Kang, Choi and Ryu (2008) showed that the breastfeeding empowerment was effective in increasing the breastfeeding rate and helped mothers to actively solve problems and cope with difficulties.

The findings showed that participants felt a sense of satisfaction and enjoyment while breastfeeding their babies. Success at breastfeeding may contribute to greater maternal role satisfaction, as a factor that might be perceived by mothers as an indication of mothering ability in general (Isabella and Isabella 1994). Successful lactation becomes an emotionally satisfying and enjoyable experience for both mother and infant, during which mother anticipates infant's needs and respond accordingly, thereby receiving positive reinforcement and validation of her role as a mother. Supporting and enabling breastfeeding practices that both satisfy the individual mother's wishes, expectations and needs, and enable mothers to derive pleasure and enjoyment from breastfeeding for themselves and their infants would help these vulnerable mothers find control, success and satisfaction, which in turn lead to empowerment in breastfeeding (Edwards 2018).

The participants in this study still holding firmly to the values and trust knowledge that is passed on to them from family members and women within their community, which includes the practice of breastfeeding. Moreover, the belief of doing and giving the best for their baby remains the key driving force for these women throughout their decision making process. In Heidari, Kohan and Keshvari's (2017) study, the participants placed special emphasis on mothers' and family members' informed beliefs in the value of breastfeeding. 
The present study showed that another aspect of empowerment in breastfeeding is receiving support from surrounding people such as mothers and husbands. Breastfeeding support was very broadly defined as "any advice, help or support with breastfeeding from any source, whether solicited or unsolicited" (Chaput et al. 2015, 306). The way that families influence breastfeeding is very diverse, depending on the make-up of the family, local culture and location (e.g., urban/rural). In some cultures, families are more supportive of breastfeeding than in others - for example, more so in Lebanon than in Ghana or Nigeria (Balogun et al. 2015). Family members, such as fathers and babies' grandmothers, are important parts of a mother's life and have a tremendous influence on breastfeeding practices. If a baby's grandmother previously breastfed, she can share her experience and knowledge and can support a mother through any challenges with breastfeeding (Grassley and Eschiti 2008). Two systematic reviews of research mostly from developing countries have demonstrated the influence of grandmothers (Aubel 2012; Negin et al. 2016). Other studies also mentioned that receiving breastfeeding support from the health care provider, family and society is an important source of emotional support during the postpartum period (Heidari, Kohan and Keshvari 2017; Nelson 2006; Di Manno, Macdonald and Knight 2015). Previous findings have also established the importance of the nurse as a source of breastfeeding information and support (Isabella and Isabella 1994).

\section{CONCLUSION}

These indigenous Temiar women were empowered in the breastfeeding practices through adequate knowledge and skills in overcoming problems and coping difficulties pertaining to breastfeeding. In addition, the family belief in the value of breastfeeding, maternal satisfaction and enjoyment in successful breastfeeding, and also support from other surrounding people helped in facilitating and strengthening the women's empowerment in breastfeeding. Empowering women especially in breastfeeding practices will help protect or restore breastfeeding cultures among the Temiar women and others all around the world. It is hoped that their experiences can be used to develop and devise comprehensive programmes with a focus on empowering women in breastfeeding and in efforts to bridge the gap of gender equality in communities, rural and urban alike. This study involved only one sub-ethnic group of the Orang Asli, the Temiar. Thus, further research is needed regarding breastfeeding practices and empowerment among other subethnic groups of the Orang Asli in Peninsular Malaysia. 


\section{ACKNOWLEDGEMENTS}

This study was made possible by the generous support of a Universiti Sains Malaysia Short Term Grant No: 304/PKANITA/61313051. We would also like to thank the Department of Orang Asli Development Malaysia for their assistance in this study.

\section{REFERENCES}

Abbass-Dick, J.M. 2013. Evaluating the effectiveness of a co-parenting breastfeeding support intervention (COSI) on exclusive breastfeeding rates at twelve weeks postpartum. PhD diss., Nursing Science, University of Toronto.

Abbass-Dick, J.M., L. Rempel, J. Rempel, T.H. Bich and D. Fisher. 2018. Breastfeeding as family teamwork: A research to practice briefing (international). The Family Initiative. April. https://familyincluded.com/breastfeeding-family-teamwork/ (accessed September 2018).

Aubel, J. 2012. The role and influence of grandmothers on child nutrition: Culturally designated advisors and caregivers. Matern Child Nutr 8(1): 19-35. https://doi .org/10.1111/j.1740-8709.2011.00333.x

Balogun, O.O., A. Dagvadorj, K.M. Anigo, E. Ota and S. Sasaki. 2015. Factors influencing breastfeeding exclusivity during the first 6 months of life in developing countries: A quantitative and qualitative systematic review. Matern Child Nutr 11(4): 433-451. https://doi.org/10.1111/men.12180

Bodley, J.H. 1994. An anthropological perspective, in cultural anthropology: Tribes, states, and the global system. http://www.wsu.edu/gened/learn-modules/top_culture/ culture-definitions/bodley-text.html (accessed August 2018).

Bazeley, P. 2007. Qualitative data analysis with NVivo. London: Sage Publications Ltd.

Chaput, K.H., C.E. Adair, A. Nettel-Aguirre, R. Musto and S.C. Tough. 2015. The experience of nursing women with breastfeeding support: A qualitative inquiry. CMAJ Open 3(3): E305-E309. https://doi.org/10.9778/cmajo.20140113

Di Manno L., J.A. Macdonald and T. Knight. 2015. The intergenerational continuity of breastfeeding intention, initiation, and duration: A systematic review. Birth 42: 5-15. https://doi.org/10.1111/birt.12148

Edwards, R. 2018. An exploration of maternal satisfaction with breastfeeding as a clinically relevant measure of breastfeeding success. Journal of Human Lactation 34(1): 93-96. https://doi.org/10.1177/0890334417722509

Grassley, J. and V. Eschiti. 2008. Grandmother breastfeeding support: what do mothers need and want? Birth 35: 329-335. https://doi.org/10.1111/j.1523-536X.2008.00260.x

Heidari, Z., M. Keshvari and S. Kohan. 2016. Clinical trial to comparison the effect of family- centered educational-supportive program on mothers' empowerment in breast-feeding. Int J Pediatr 4(3): 1445-1451. 
Heidari, Z., S. Kohan and M. Keshvari. 2017. Empowerment in breastfeeding as viewed by women: A qualitative study. J Educ Health Promot 6: 33. https://doi.org/10.4103/ jehp.jehp_34_16

Isabella, P.H. and R.A. Isabella. 1994. Correlates of successful breastfeeding: A study of social and personal factors. Journal of Human Lactation 10(4): 257-264. https://doi.org/10.1177/089033449401000421

Kang, J.S., S.Y. Choi and E.J. Ryu. 2008. Effects of a breastfeeding empowerment programme on Korean breastfeeding mothers: A quasi-experimental study. Int J Nurs Stud 45(0): 14-23. https://doi.org/10.1016/j.ijnurstu.2007.03.007

Ketabi, M., B. Yazdkhasti and Z. Farohki. 2003. Empowering women to participate in the development. Women's Research 1(7): 5-30.

Kohan, S., Z. Heidari and M. Keshvari. 2016. Facilitators for empowering women in breastfeeding: A qualitative study. Int J Pediatr 4(1): 1287-1296.

Mitchell-Box, K.M. and K.L. Braun. 2013. Impact of male-partner-focused interventions on breastfeeding initiation, exclusivity, and continuation. J Hum Lact 29(4): 473479. https://doi.org/10.1177/0890334413491833

Morris, A., H. Love, Z. van Aar, C. Liles and C. Roskell. 2018. Integrating renal nutrition guidelines into daily family life: A qualitative exploration. J Hum Nutr Diet 31: 3-11. https://doi.org/10.1111/jhn.12483

Munhall, L.P. 2011. Nursing research: A qualitative perspective. London: Jones and Bartlett Co.

Negin, J., J. Coffman, P. Vizintin and C. Raynes-Greenow. 2016. The influence of grandmothers on breastfeeding rates: a systematic review. BMC Pregnancy and Childbirth 16: 91. https://doi.org/10.1186/s12884-016-0880-5

Nelson, A.M. 2006. A metasynthesis of qualitative breastfeeding studies. J Midwifery Womens Health 51: 13-20. https://doi.org/10.1016/j.jmwh.2005.09.011

Omer-Salim, A. 2018. In bridging the gap of gender equality in rural communities through breastfeeding. Here's how. http:/waba.org.my/blog/bridging-gap-gender -equality-rural-communities-breastfeeding-heres/ (accessed September 2018).

Van Esterik, P. n.d. Breastfeeding: A feminist issue. https://www.waba.org.my/resources/ activitysheet/acsh4.htm (accessed September 2018).

World Alliance for Breastfeeding Action. 1995. Breastfeeding and empowerment. http://www.worldbreastfeedingweek.net/support/1995/af\%2795_eng3.pdf (accessed August 2018).

World Health Organization (WHO). 2001. The world health organization's infant feeding recommendation. http:/www.who.int/nutrition/topics/infantfeeding recommendation/en/index.html (accessed August 2018).

Yang, X., L. Gao, W. Ip and W.C.S. Chan. 2016. Predictors of breastfeeding self-efficacy in the immediate postpartum period: A cross-sectional study. Midwifery 41: 1-8. https://doi.org/10.1016/j.midw.2016.07.011 